\title{
Computer Simulation of Noise Effects of the Neighborhood of Stimulus Threshold for a Mathematical Model of Homeostatic Regulation of Sleep-Wake Cycles
}

\author{
Wuyin Jin, ${ }^{1}$ Qian Lin, ${ }^{2}$ An Wang, ${ }^{1}$ and Chunni Wang ${ }^{3}$ \\ ${ }^{1}$ School of Mechanical \& Electronical Engineering, Lanzhou University of Technology, Lanzhou 730050, China \\ ${ }^{2}$ Editorial Department of Journal of Lanzhou University of Technology, Lanzhou University of Technology, Lanzhou 730050, China \\ ${ }^{3}$ Department of Physics, Lanzhou University of Technology, Lanzhou 730050, China \\ Correspondence should be addressed to Wuyin Jin; wuyinjin@hotmail.com
}

Received 4 July 2017; Accepted 13 September 2017; Published 22 October 2017

Academic Editor: Sajad Jafari

Copyright ( 2017 Wuyin Jin et al. This is an open access article distributed under the Creative Commons Attribution License, which permits unrestricted use, distribution, and reproduction in any medium, provided the original work is properly cited.

\begin{abstract}
The noise effects on a homeostatic regulation of sleep-wake cycles' neuronal mathematical model determined by the hypocretin/orexin and the local glutamate interneurons spatiotemporal behaviors are studied within the neighborhood of stimulus threshold in this work; the neuronal noise added to the stimulus, the conductance, and the activation variable of the modulation function are investigated, respectively, based on a circadian input skewed in sine function. The computer simulation results suggested that the increased amplitude of external current input will lead to the fact that awakening time is advanced but the sleepy time remains the same; for the bigger conductance and modulation noise, the regulatory mechanism of the model sometimes will be collapsed and the coupled two neurons of the model show very irregular activities; the falling asleep or wake transform appears at nondeterminate time.
\end{abstract}

\section{Introduction}

Noise not only is a problem for neurons, but also can be a solution in information processing. Several strategies have been adopted to use noise in this fashion; for example, stochastic resonance is a process by which the ability of threshold-like systems to detect and transmit weak signals can be enhanced by the presence of a certain level of noise [1]. Neural noise is a general term that designates random influences on the transmembrane voltage of single neurons and by extension of the firing activity of neural networks. This noise can influence the transmission and integration of signals from other neurons and alter the firing activity of neurons in isolation [2-4], and there are some significant effects near bifurcation points $[5,6]$, the weak neural noise, that seem to be less relevant when the neurons operate in spike generating regime for a suprathreshold; however, the situation is completely different in the neighborhood of threshold where noise can induce significant changes in the impulse patterns; furthermore, in the central neural system, the neurons often work in the neighborhood of threshold, but neurons are heterogeneous and noise is inevitable [7]. Sleep is essential for the maintenance of the brain and the body, yet many features of sleep are poorly understood and mathematical models are an important tool for probing proposed biological mechanisms; in addition, noise is an inevitable factor in real neuronal systems, which plays an important role in spatiotemporal dynamics of neuronal networks, for nearly a century of study; some regulation nonlinear sleep models about circadian [8], diversity-induced resonance [9], temporal dynamics [10], physiological substrates [11, 12], and more have been proposed to investigate the neural regulatory mechanism for sleep-wake cycle; however, sleep and its underlying processes still hold many mysteries; it remains unclear how identified brain regions interact to bring about the different stages of sleep and wakefulness, how the timing of sleep depends on the length of time spent awake and work load, and how pathologies associated with sleep, such as narcolepsy, arise [13]. As we all know, there are homeostatic formation mechanism and biological function 
between connection neurons $[14,15]$; in this work, we study the spatiotemporal behaviors of noise effects in the neighborhood of stimulus threshold for a mathematical model of homeostatic regulation of sleep-wake cycles proposed by Postnova et al. [16]. The effects of neuronal noise added to the stimulus, the conductance, and the modulation function are investigated, respectively, based on a circadian input skewed in sine function proposed by Daan et al. [17].

\section{Model and Input}

The mathematical model of regulation sleep-wake cycles employed in this work is suggested by Postnova et al., the homeostatic regulation process in this model is determined by the neuropeptide hypocretin/orexin (Hcrt/ox), and the Hcrt/ox neurons are silent during sleep and active during wakefulness depending on reciprocal excitatory synaptic connections with local glutamate (Glu) interneurons. The model formed by two simplified Hodgkin-Huxley type neurons that are connected via Glu synapses, one of which additionally contains Hcrt/ox as the functionally relevant cotransmitter (see Figure 1 of [16]); particularly, they proposed a novel modulation function for the synaptic efficacy to regulate Hcrt/ox neuronal firing and silent state [16], and the important role of the orexin also is studied by Rempe et al. [13]. The mathematical model of regulation of sleep-wake cycles is given in the following form [16]; the membrane of two coupled neurons is given by

$$
\begin{aligned}
C \frac{d V_{\mathrm{Glu}}}{d t}= & -I_{\mathrm{lGlu}}-I_{\mathrm{NaGlu}}-I_{\mathrm{KGlu}}-I_{\text {sys } \mathrm{Hcrt} / \text { Orx }} \\
C \frac{d V_{\mathrm{Hcrt} / \text { Orx }}}{d t}= & -I_{\mathrm{lHcrt} / \mathrm{Orx}}-I_{\mathrm{NaHcrt} / \text { Orx }}-I_{\mathrm{KHcrt} / \text { Orx }} \\
& -I_{\text {sys }_{\mathrm{Glu}}}+I_{\mathrm{ext}},
\end{aligned}
$$

where the leak, sodium, and potassium currents are described by

$$
\begin{aligned}
I_{1_{n}} & =g_{1}\left(V_{n}-E_{1}\right), \\
I_{\mathrm{Na}_{n}} & =g_{\mathrm{Na}} \alpha_{\mathrm{Na}_{n}}\left(V_{n}-E_{\mathrm{Na}}\right), \\
I_{\mathrm{K}_{n}} & =g_{\mathrm{K}} \alpha_{\mathrm{K}_{n}}\left(V_{n}-E_{\mathrm{K}}\right) ;
\end{aligned}
$$

here, $n$ refers to Hcrt/ox and Glu, respectively. The sodium current is considered to be activated instantaneously; the potassium current is activated with a time delay

$$
\begin{aligned}
\frac{d a_{\mathrm{K} n}}{d t} & =\frac{a_{\mathrm{K} n \infty}-a_{\mathrm{Kn}}}{\tau_{\mathrm{K}}}, \\
a_{\mathrm{K} n \infty} & =\frac{1}{1+\exp \left(-s_{\mathrm{K}}\left(V_{n}-V_{0 \mathrm{~K}}\right)\right)}, \\
a_{\mathrm{Na}} & =a_{\mathrm{Nan} \infty}=\frac{1}{1+\exp \left(-s_{\mathrm{Na}}\left(V_{n}-V_{0 \mathrm{Na}}\right)\right)} .
\end{aligned}
$$

And the transmembrane currents

$$
I_{\text {syn }_{\text {glu }}}=g_{\text {glu }} a_{\text {glu }}\left(V_{\text {Hcrt/Orx }}-E_{\text {syn }}\right),
$$

with

$$
\begin{aligned}
& \frac{d a_{\mathrm{glu}}}{d t}=\frac{a_{\mathrm{glu} \infty}-a_{\mathrm{glu}}}{\tau_{\mathrm{glu}}}, \\
& a_{\text {glu } \infty}=\frac{1}{1+\exp \left(-s_{\text {syn }}\left(V_{\text {Glu }}-V_{\text {spike }}\right)\right)} \text {, } \\
& I_{\text {syn }_{\text {Hcrt/orx }}}=I_{\text {glu(Hcrt/Orx })}+I_{\text {Hcrt/Orx }} \text {, } \\
& I_{\mathrm{glu}(\mathrm{Hcrt} / \mathrm{Orx})}=g_{\mathrm{glu}(\mathrm{Hct} / \mathrm{Orx})} a_{\mathrm{glu}(\mathrm{Hcrt} / \mathrm{Orx})}\left(V_{\mathrm{Glu}}-E_{\mathrm{syn}}\right) \text {, } \\
& I_{\mathrm{Hcrt} / \mathrm{Orx}}=g_{\mathrm{glu}(\mathrm{Hcrt} / \mathrm{Orx})} a_{\mathrm{Hcrt} / \mathrm{Orx}}\left(V_{\mathrm{Glu}}-E_{\mathrm{syn}}\right),
\end{aligned}
$$

which are modelled by

$$
\begin{aligned}
\frac{d a_{\mathrm{glu}(\mathrm{Hcrt} / \mathrm{Orx})}}{d t} & =\frac{a_{\mathrm{Hcrt} / \mathrm{Orx} \infty}-a_{\mathrm{glu}(\mathrm{Hcrt} / \mathrm{Orx})}}{\tau_{\mathrm{Glu}}}, \\
\frac{d a_{\mathrm{Hcrt} / \mathrm{Orx}}}{d t} & =\frac{M a_{\mathrm{Hcrt} / \text { Orx } \infty}-a_{\mathrm{Hcrt} / \text { Orx }}}{\tau_{\mathrm{Hcrt} / \mathrm{Orx}}}, \\
a_{\mathrm{Hcrt} / \text { Orxuo }} & =\frac{1}{1+\exp \left(-s_{\text {syn }}\left(V_{\text {Hcrt/Orx }}-V_{\text {spike }}\right)\right)},
\end{aligned}
$$

with a homeostatic function defined by

$$
\frac{d M}{d t}=\frac{M_{\max }-M}{\tau_{\text {increase }}}-\frac{M a_{\mathrm{Hcrt} / \text { Orxo }}}{\tau_{\text {decrese }}} .
$$

All the parameters' meaning and value of (1)-(10) are in detail described in [16], as well as their supplements.

The external current input is applied to the Hcrt/ox neuron as a circadian input proposed in [17], which is defined by skewed sine function; an additional phase $\operatorname{shift} \varphi$ is added to be consistent with day and night

$$
\begin{aligned}
I_{\text {ext }} & =A[0.97 \sin (\omega t-\varphi)+0.22 \sin (2 \omega t-\varphi) \\
& +0.07 \sin (3 \omega t-\varphi)+0.03 \sin (4 \omega t-\varphi) \\
& +0.001 \sin (5 \omega t-\varphi)+1],
\end{aligned}
$$

where $A$ is a parameter for scaling the amplitude of the stimulus, $\omega$ is the angular frequency of the one-day-night circadian current, and $\varphi=\pi / 3$ is an additional phase shift in this work.

\section{Computer Simulation of Noise Effects of Regulation of Sleep-Wake Cycles}

3.1. Regulation of Sleep-Wake Cycles of the Deterministic Model. As an illustration for the mathematical model of homeostatic regulation of sleep-wake cycles, the coupled Hcrt/ox and Glu of sleep-wake cycles activities are shown in Figures 1(b) and 1(c), respectively, under one typical amplitude of external current input $A=1.0 \mu \mathrm{A} / \mathrm{cm}^{2}$ (shown in Figure 1(a)); these neuronal activities are ongoing with regulation function $M$ (shown in Figure 1(d)). Seen from Figure 1, the presynaptic Hcrt/ox neuron induces firing (waking 


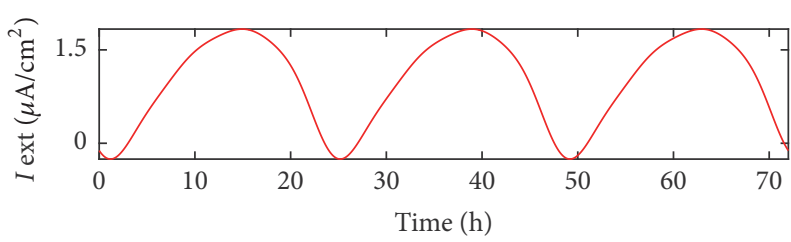

(a)

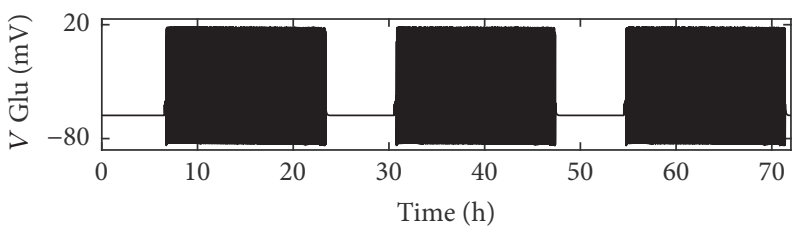

(c)

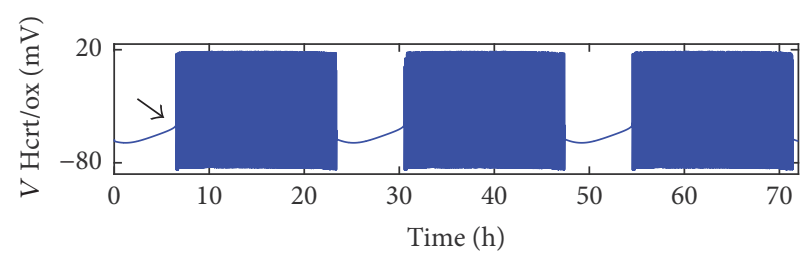

(b)

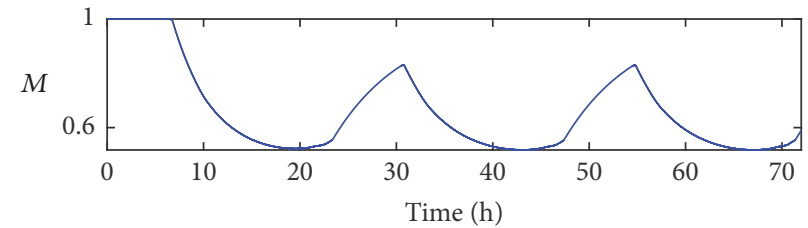

(d)

FIGURE 1: An illustration of the mathematical model of homeostatic regulation of sleep-wake cycles, the coupled Hcrt/ox (b), and Glu (c) of sleep-wake cycles activities, respectively, under one typical amplitude of external current input $A=1.0 \mu \mathrm{A} / \mathrm{cm}^{2}$ (a), and the variety of regulation function $M(\mathrm{~d})$. Moreover, the increased amplitude of external current input will advance wake time but slightly change sleepy time.

up) almost at half past six (marked by an arrow in Figure 1(b)) in the morning and recovers to the silence (falling asleep) at half past twenty-three at midnight; these results are consistent with physiological time. The postsynaptic spikes appear with a certain delay because Hcrt/ox postsynaptic potentials need time for sufficient superposition that activates neuron. The regulation function $M$ declines with beginning spike and recovers when also the presynaptic firing is switched off.

One more interesting result we got in this work is that the increased amplitude of external current input will lead to awakening time being advanced; for example, the awakening time will be advanced from half past six to half past three in the morning while the amplitude $A$ of external current input increased from $1.0 \mu \mathrm{A} / \mathrm{cm}^{2}$ to $12 \mu \mathrm{A} / \mathrm{cm}^{2}$, but the sleepy time still stays at half past twenty-three at midnight; this phenomenon may be like the fact that the older people's sleep becomes less and less.

3.2. Effects of Noise on Regulation of Sleep-Wake Cycles. Based on research of deterministic regulation of sleep-wake cycles model, we found that, to excite the coupled Hcrt/ox and Glu of the sleep-wake cycles model neuron, the external stimulus current amplitude must be greater than $0.465 \mu \mathrm{A} / \mathrm{cm}^{2}$; therefore, in this section, the amplitude of external current input is set to $A=0.46 \mu \mathrm{A} / \mathrm{cm}^{2}$ as a stimulus threshold only can excite Hcrt/ox neuron in short duration, but the Glu neuron just displays the subthreshold activity shown in Figure 2(a).

And, an additive Gaussian white noise is added to current, conductance, and regulation function, respectively, to compare study significant differences in the regulation process; here, the Gaussian white noise $\xi$ is calculated according to

$$
\xi=\left[\left(-\frac{4 D}{\Delta t}\right) \ln (a)\right]^{1 / 2} \cos (2 \pi b),
$$

where $\Delta t$ is the time step of the integrator and $a, b \in[0,1]$ are uniformly distributed random numbers. The noise intensity is adjusted by the parameter $D$.
3.2.1. Effects of Current Noise. When the amplitude of external current input locates within subthreshold regime, the regulation of sleep-wake cycles function cannot work normally; for example, $A=0.46 \mu \mathrm{A} / \mathrm{cm}^{2}$, as subthreshold external input can only excite Hcrt/ox neuron in short duration one time a day in the noonday, as shown in Figure 2(a), but the Glu neuron just displays the subthreshold activity without any fire; all of these results in the regulation function $M$ generate small vibration, which could be found in the bottom line of Figure 2(a).

With the additional noise defined in (12), being added to the right of (2) as current noise, the model produces general regulated activation while the value of noise intensity $(D)$ is greater than a certain value even if the amplitude of external current input locates within subthreshold regime, as an example, when $A=0.46 \mu \mathrm{A} / \mathrm{cm}^{2}$ and $D \geq 0.007$, for the other smaller intensity noise in neighborhood, leading to the seemingly random regulation process (as shown in Figure 2(b), where $D=0.006$ ); occasionally, the model shows a sudden and terrible change; it could be seen in Figure 2(c) with same noise intensity $D=0.006$ too; the Glu neuron does not respond (fire) to Hcrt/ox neuron on the third cycle, which looks like falling ill.

3.2.2. Effects of Conductance Noise. For an evaluation of the efficacy of noise in the different conductance, we firstly have examined the noise effects on the slow repolarizing variable of two coupled neurons (Hcrt/ox and Glu); two different noises with the same intensity as defined in (12) are added to the right of (4) at the same time; the amplitude of external current input is still set to $A=0.46 \mu \mathrm{A} / \mathrm{cm}^{2}$ as subthreshold stimulus. The simulation results demonstrate that both of the neurons of sleep-wake cycle model are excited (wakefulness) all the time without sleep when noise intensity $D>0.00002$, seeming to be losing sleep.

While $D \in[0.0000001,0.00001]$, the model generates similar regulation process; especially when $D$ is close to 

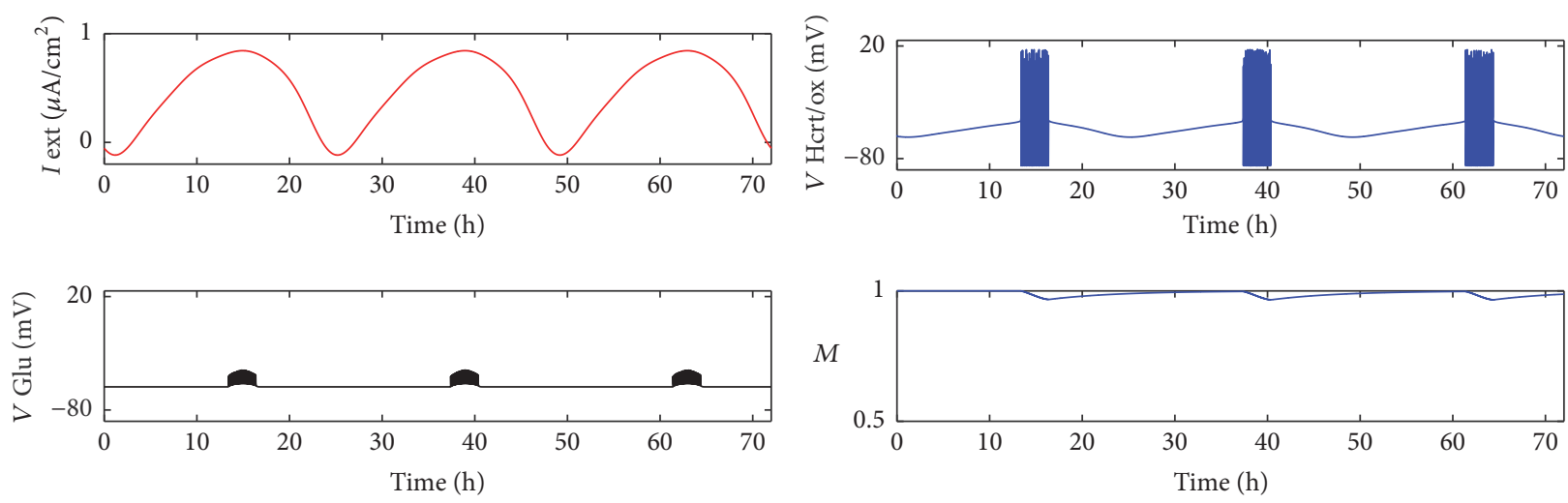

(a)
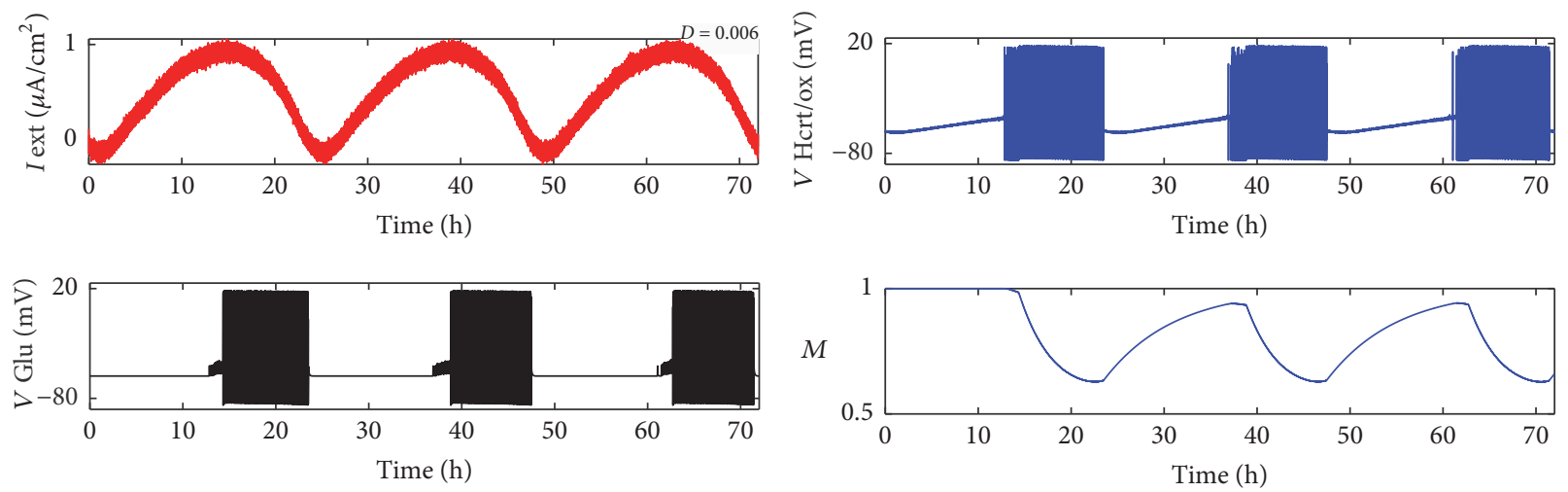

(b)
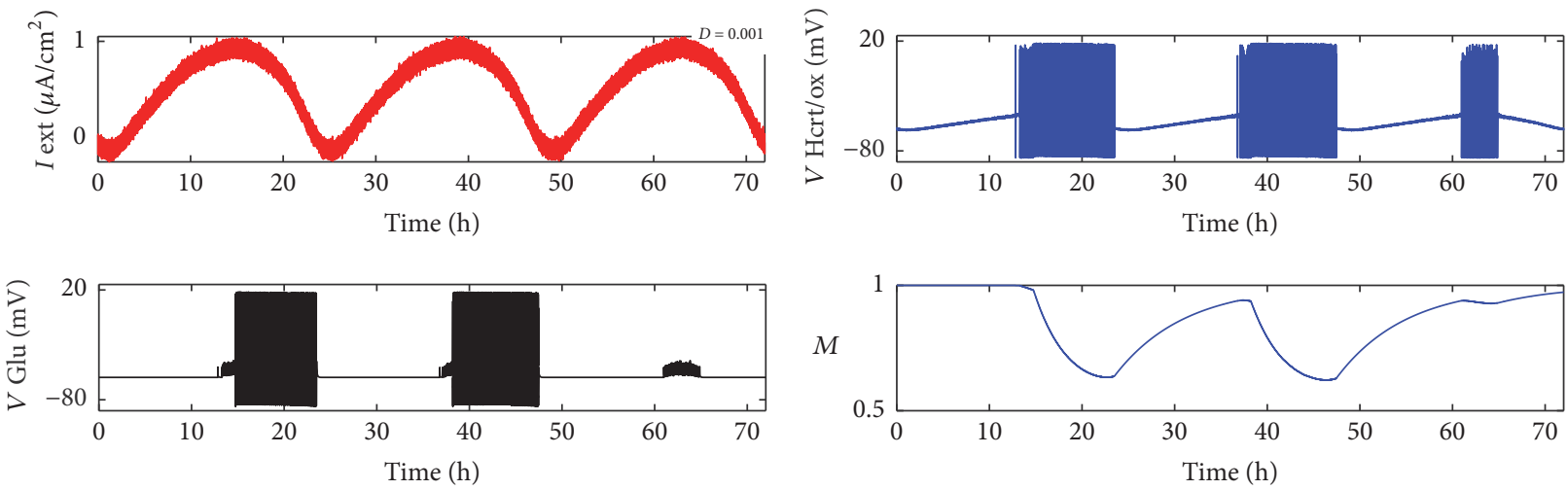

(c)

FiguRE 2: In subthreshold regime, the amplitude of external current input is set to $A=0.46 \mu \mathrm{A} / \mathrm{cm}^{2}$ to the deterministic regulation of sleepwake cycles model stimulus which only can excite Hcrt/ox neuron, but the Glu neuron just displays the subthreshold activities (a), but, after an additive Gaussian white noise is added to current, leading to the seemingly random regulation process, it sometimes shows similar regulation function (b) (where the noise intensity $D=0.006)$; occasionally, the model shows a sudden and terrible change (c) $($ where $D=0.006$ too).

0.00001, the coupled two neurons can wake up (fire) about half past six in the morning and become silent (sleepy) about half past twenty-two at midnight, shown in Figure 3(a), which are similar to those in Figure 1. But, with the decreasing noise intensity from 0.00001 to 0.0000001 , the wake time will be delayed more and more with the decrease of intensity changing from about six o'clock in the morning to two o'clock in the noonday; however, the sleepy time almost does not change, staying at about half past twenty-two at midnight.

For the case of continued decrease of noise strength below 0.0000001 , the similar regulation function will be broken like behavior in Figure 2(c).

Moreover, the effects of individual noise also have been examined separately; for the same strength noise, the Hcrt/ox 

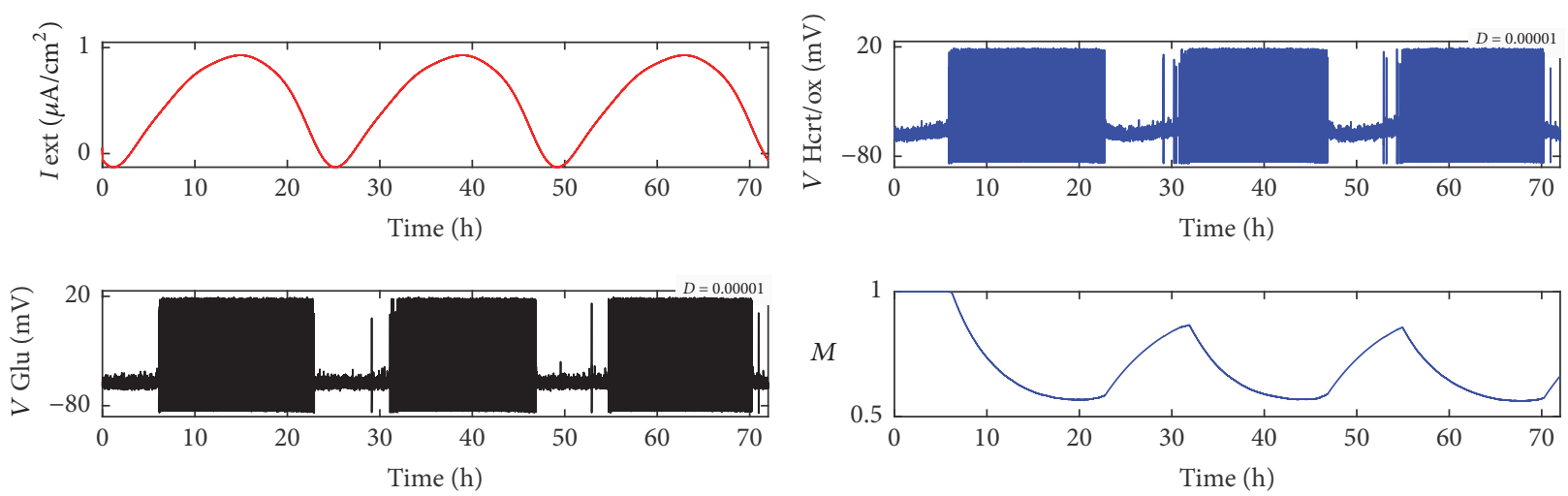

(a)
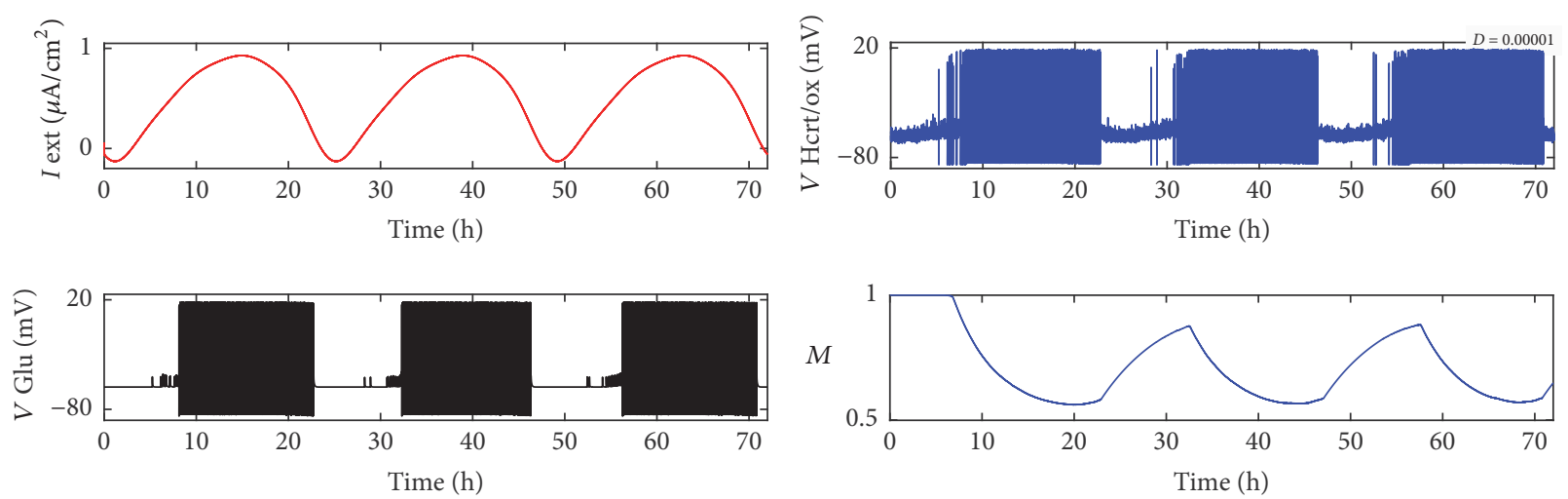

(b)
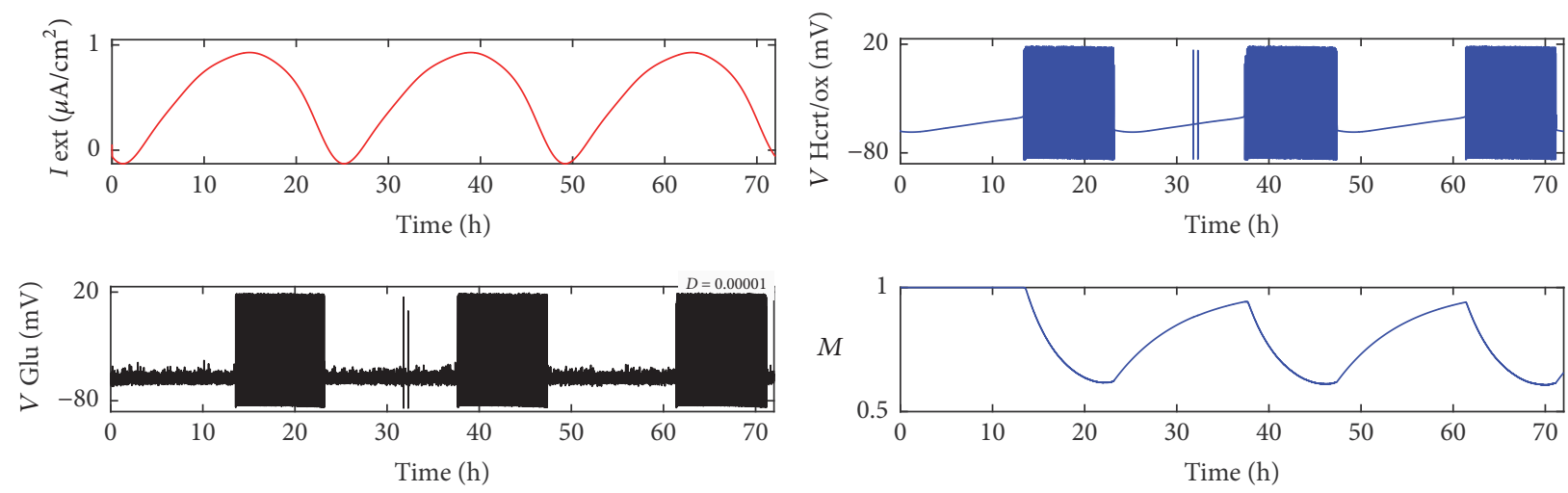

(c)

Figure 3: Effects of conductance noise. With the amplitude of external current input still being set to $A=0.46 \mu \mathrm{A} / \mathrm{cm}^{2}$ as subthreshold stimulus, the proper noise added to the slow repolarizing variable of two coupled Hcrt/ox and Glu neuron can produce the similar regulation function (a). For the individual noise, the Hcrt/ox neuron's conductance noise generates more significant effect on the regulation function (b) than the Glu neuron (c). Furthermore, the neurons of sleep-wake cycle model are excited all the time without sleep when noise intensity $D>0.00002$.

neuron conductance noise generates more significant effect on the regulation function than the Glu neuron, which could be found in Figures 3(b) and 3(c).

3.2.3. Effects of the Modulation Function. To demonstrate the effects of noise modulation function, the same amplitude of external current input $A=0.46 \mu \mathrm{A} / \mathrm{cm}^{2}$ is chosen as subthreshold stimulus; through the study of different noise, we found that the regulation process of modulation function almost does not change under the weak noise, as shown in Figure 4(a) for $D=0.000000001$; the neurons' activity mainly depends on the external current input. For the bigger strength of noise, the function of regulation will lose its effectiveness (see Figure 4(b) for $D=0.0000001$ ) with increasing noise intensity, even damaging the function of the neurons themselves, as an example shown in Figure 4(c) for $D=0.01$; the value $(M)$ of the modulation function randomly changes from -40 to 20 , obviously different from 

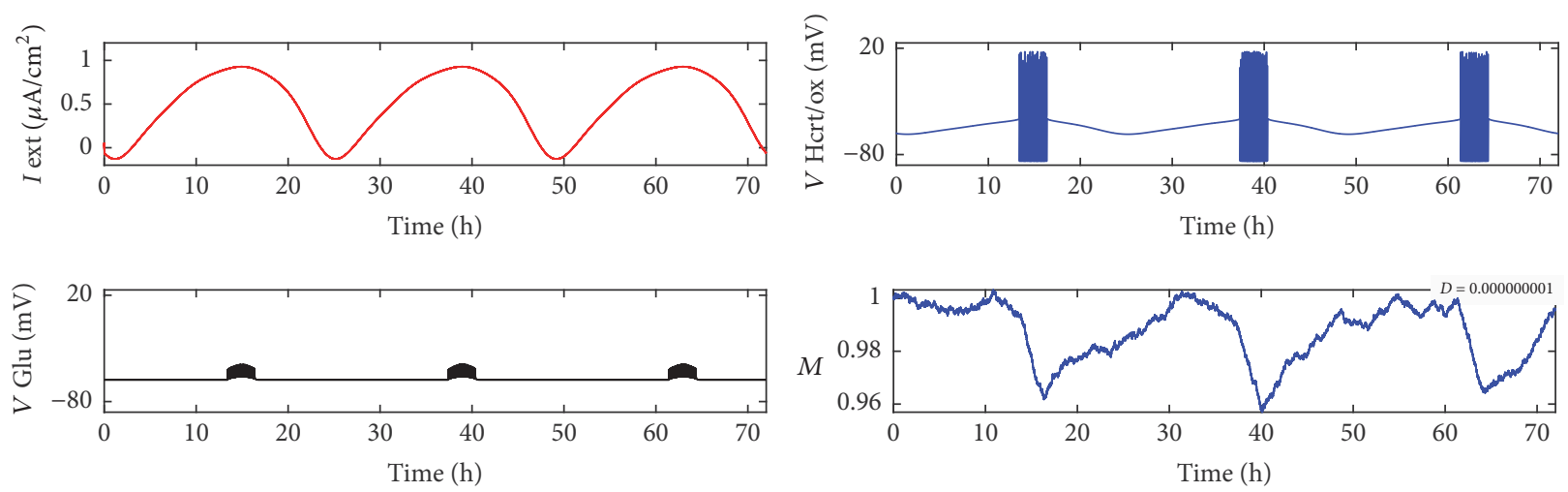

(a)
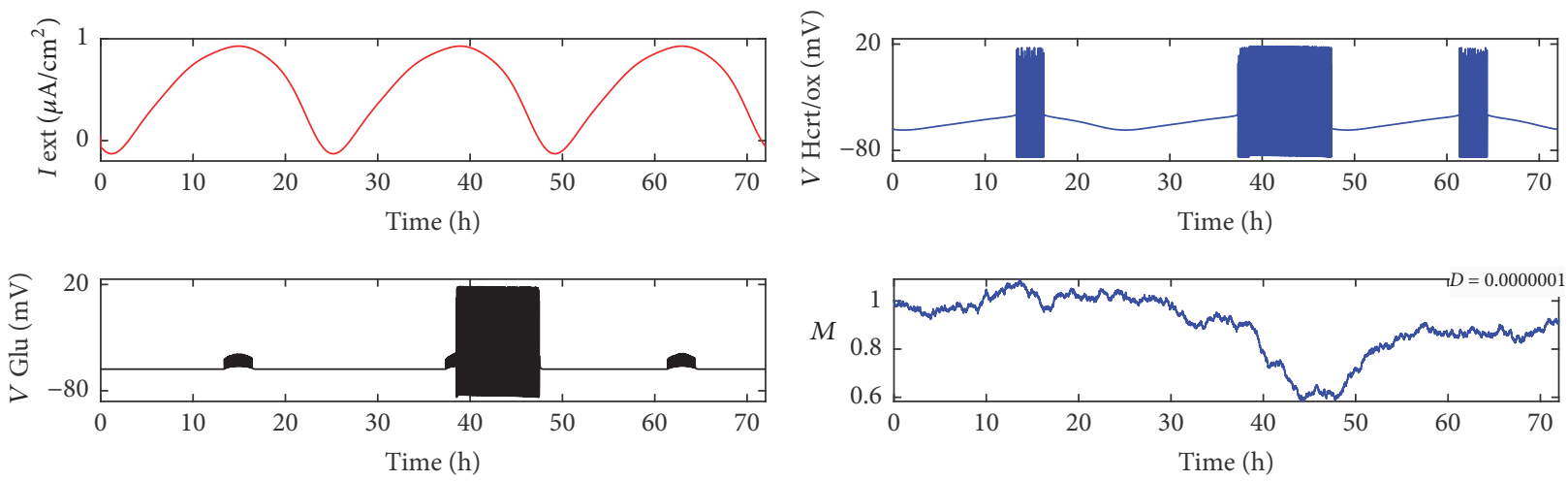

(b)
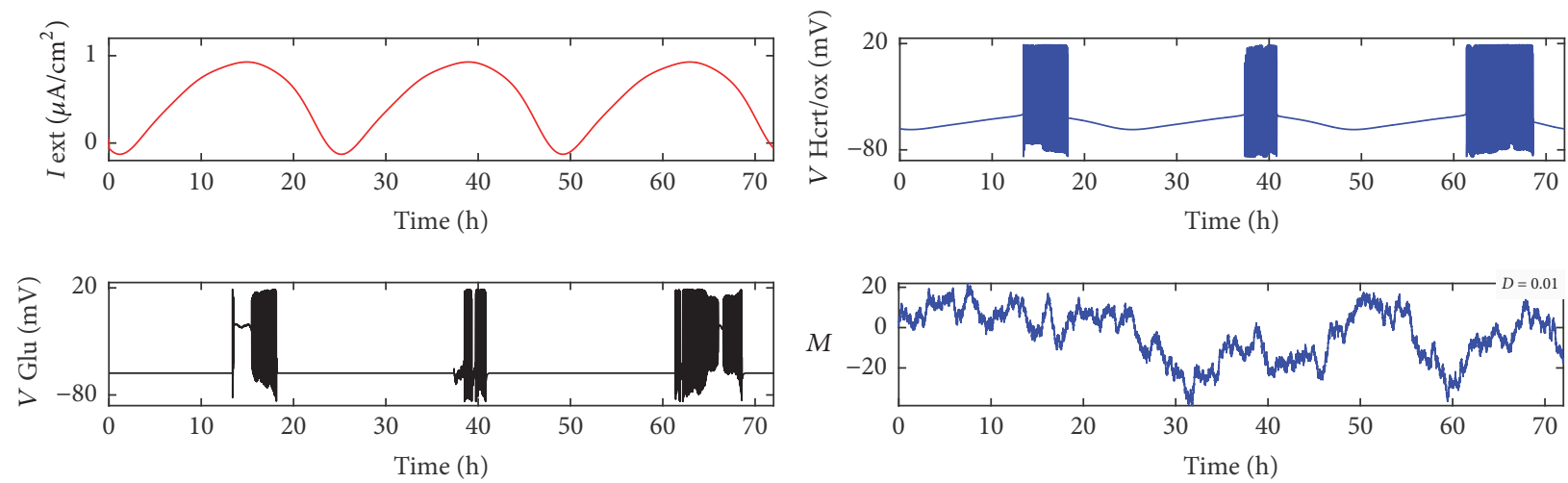

(c)

FIGURE 4: With the same subthreshold amplitude of external current input $A=0.46 \mu \mathrm{A} / \mathrm{cm}^{2}$, the small noise intensity of the modulation function has weak effect on the regulation process, as shown in (a) for $D=0.000000001$, but, for the bigger strength of noise, the function of regulation will lose its effectiveness ( $(b)$ for $D=0.0000001)$, even damaging the function of the neurons themselves; the value of the modulation function becomes very bigger and randomly changes within $-40 \sim 20$ (c), all of which means that homeostatic regulation function of sleep-wake cycles is more sensitive to noise.

normal (see Figure 1), and the Glu neuron is depolarized for a long duration, meaning that it is dead from the biological point of view.

\section{Conclusions}

The positive and negative function of noise in the neighborhood of stimulus threshold for a mathematical model of homeostatic regulation of sleep-wake cycles are studied based on a circadian input; the noise is added to the stimulus, the conductance, and the modulation function; the results illustrated that the weak current, conductance, and variable of regulation function noise have significant influence on the transitions between sleep and wakefulness, the bigger amplitude of external current will advance wake time but slightly change sleepy time, and the weak conductance and modulation noise generate seemingly regulation process, but, for the strong noise, the regulation function will lose its 
effectiveness and even damage the function of the neurons themselves.

\section{Conflicts of Interest}

The authors declare that they have no conflicts of interest.

\section{Acknowledgments}

The authors are grateful for the support of the National Natural Science Foundation of China under Grant nos. 11372122 and 11072099.

\section{References}

[1] A. A. Faisal, L. P. J. Selen, and D. M. Wolpert, "Noise in the nervous system," Nature Reviews Neuroscience, vol. 9, no. 4, pp. 292-303, 2008.

[2] A. Longtin, "Neuronal noise," Scholarpedia, vol. 8, no. 9, p. 1618, 2007.

[3] G. Wang, W. Jin, and C. Hu, "The complete synchronization of Morris-Lecar neurons influenced by noise," Nonlinear Dynamics, vol. 73, no. 3, pp. 1715-1719, 2013.

[4] G. Wang, W. Jin, and A. Wang, "Synchronous firing patterns and transitions in small-world neuronal network," Nonlinear Dynamics, vol. 81, no. 3, pp. 1453-1458, 2015.

[5] B. Jia and H. G. Gu, "Dynamics and physiological roles of stochastic firing patterns near bifurcation points," International Journal of Bifurcation \& Chaos, vol. 27, no. 7, Article ID 1750113, 2017.

[6] H. Gu, Z. Zhao, B. Jia, and S. Chen, "Dynamics of on-off neural firing patterns and stochastic effects near a sub-critical Hopf bifurcation," PLoS ONE, vol. 10, no. 4, Article ID e0121028, 2015.

[7] S. Postnova, C. Finke, W. Jin, H. Schneider, and H. A. Braun, "A computational study of the interdependencies between neuronal impulse pattern, noise effects and synchronization," Journal of Physiology-Paris, vol. 104, no. 3-4, pp. 176-189, 2010.

[8] S. Postnova, S. W. Lockley, and P. A. Robinson, "Sleep propensity under forced desynchrony in a model of arousal state dynamics," Journal of Biological Rhythms, vol. 31, no. 5, pp. 498508, 2016.

[9] M. Patriarca, S. Postnova, H. A. Braun, E. Hernández-García, and R. Toral, "Diversity and noise effects in a model of homeostatic regulation of the sleep-wake cycle," PLoS Computational Biology, vol. 8, no. 8, Article ID e1002650, e1002650, 17 pages, 2012.

[10] P. McCauley, L. V. Kalachev, D. J. Mollicone, S. Banks, D. F. Dinges, and H. P. A. Van Dongen, "Dynamic circadian modulation in a biomathematical model for the effects of sleep and sleep loss on waking neurobehavioral performance," SLEEP, vol. 36, no. 12, pp. 1987-1997, 2013.

[11] M. Fleshner, V. Booth, D. B. Forger, and C. G. Diniz Behn, "Circadian regulation of sleep-wake behaviour in nocturnal rats requires multiple signals from suprachiasmatic nucleus," Philosophical Transactions of the Royal Society A: Mathematical, Physical \& Engineering Sciences, vol. 369, no. 1952, pp. 38553883, 2011.

[12] S. Sorooshyari, R. Huerta, and L. De Lecea, "A framework for quantitative modeling of neural circuits involved in sleep-towake transition," Frontiers in Neurology, vol. 6, article no. 32, 2015.
[13] M. J. Rempe, J. Best, and D. Terman, "A mathematical model of the sleep/wake cycle," Journal of Mathematical Biology, vol. 60, no. 5, pp. 615-644, 2010.

[14] C. Wang, S. Guo, and Y. Xu, "Formation of autapse connected to neuron and its biological function," Complexity, vol. 2017, Article ID 5436737, 9 pages, 2017.

[15] M. S. Goldman, "Memory without Feedback in a Neural Network," Neuron, vol. 61, no. 4, pp. 621-634, 2009.

[16] S. Postnova, K. Voigt, and H. A. Braun, "A mathematical model of homeostatic regulation of sleep-wake cycles by hypocretin/ orexin," Journal of Biological Rhythms, vol. 24, no. 6, pp. 523535, 2009.

[17] S. Daan, D. G. Beersma, and A. A. Borbély, "Timing of human sleep: recovery process gated by a circadian pacemaker," American Journal of Physiology-Endocrinology and Metabolism, vol. 246, no. 2, pp. R161-R183, 1984. 


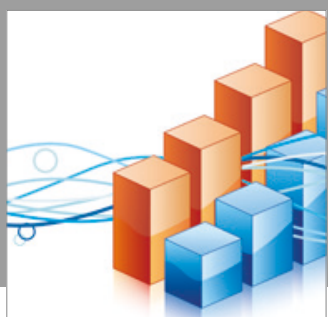

Advances in

Operations Research

vatersals

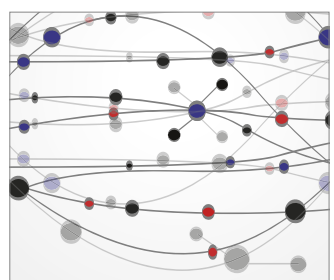

\section{The Scientific} World Journal
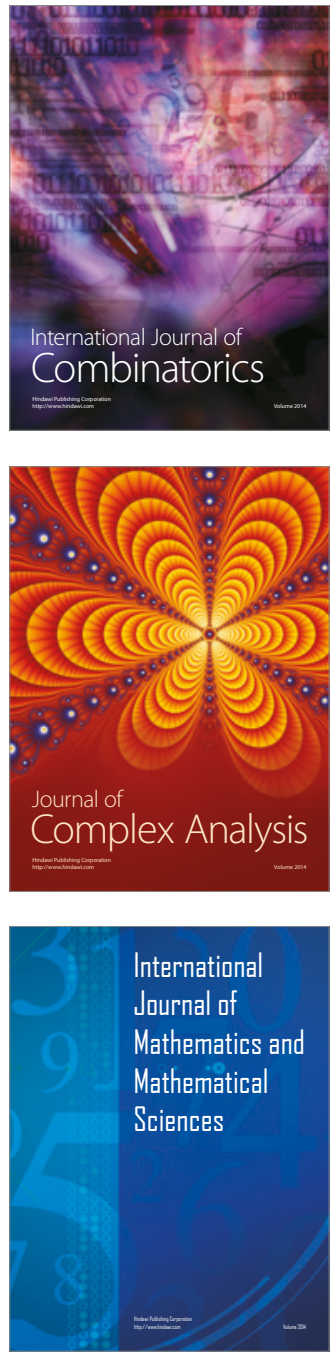
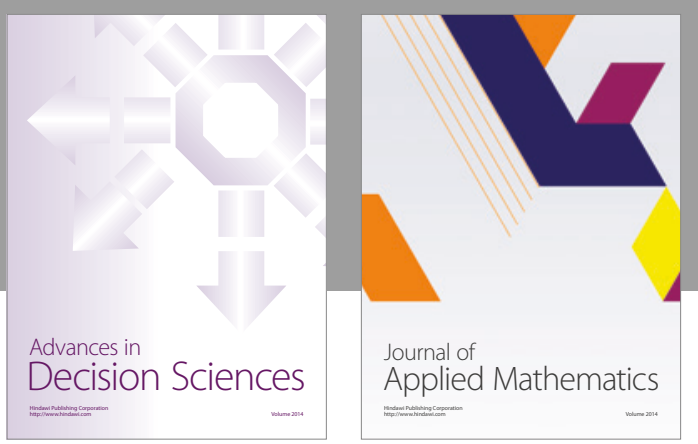

Algebra

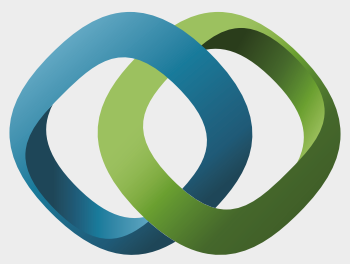

\section{Hindawi}

Submit your manuscripts at

https://www.hindawi.com
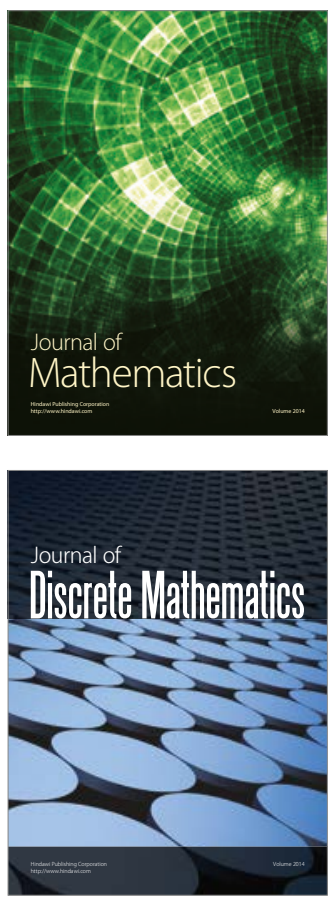

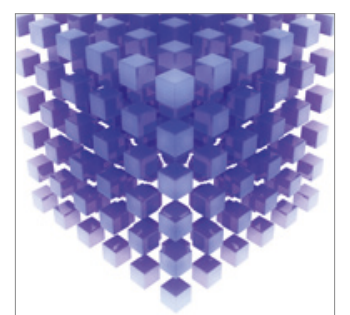

Mathematical Problems in Engineering
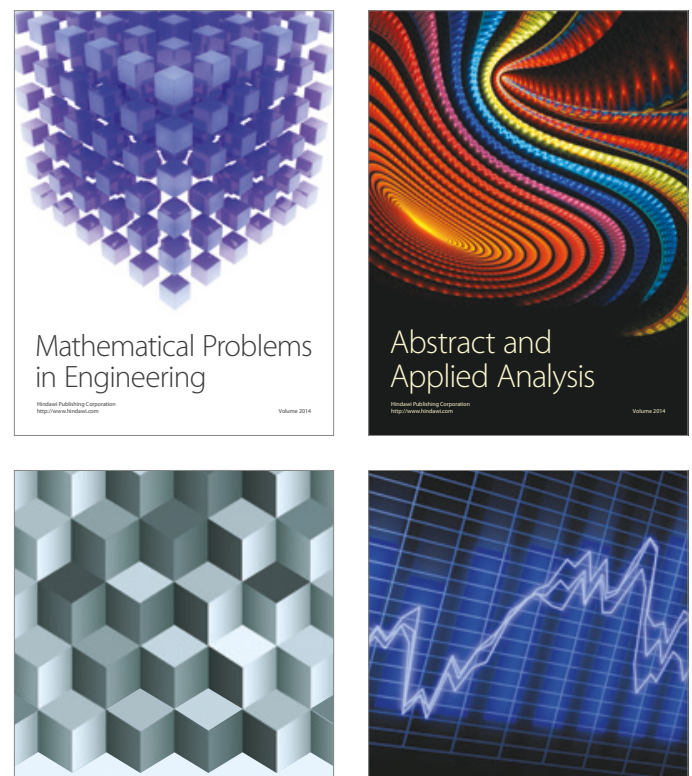

Journal of

Function Spaces

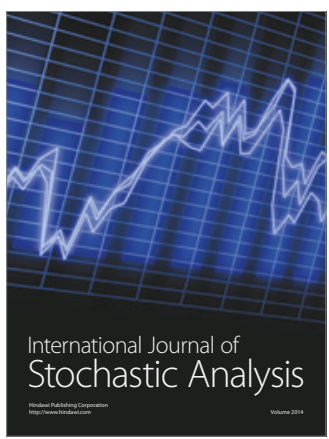

Probability and Statistics
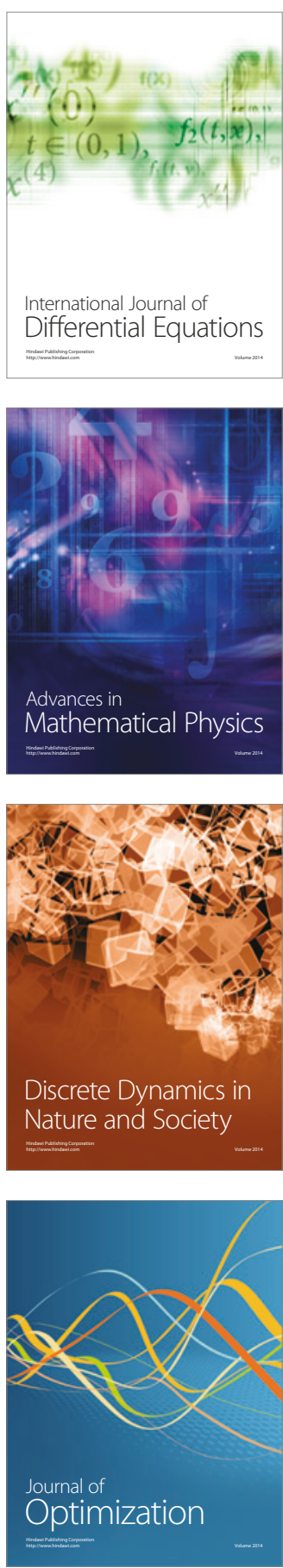